\title{
EFICIÊNCIA DE FUNGICIDAS SISTÊMICOS NO CONTROLE DA FERRUGEM DO EUCALYPTUS
}

\author{
Edival Ângelo Valverde Zauza ${ }^{2}$, Michele Margarido Fonseca Couto², Luiz Ântonio Maffia² e Acelino \\ Couto Alfenas ${ }^{2}$
}

\begin{abstract}
RESUMO - Avaliaram-se os efeitos curativo e protetor e o tempo de absorção de fungicidas sistêmicos no controle da ferrugem do eucalipto. Azoxyztrobin, triadimenol, tetraconazole, tebuconazole e epoxiconazole + pyraclostrobin apresentaram $100 \%$ de ação curativa quando aplicados até quatro dias após a inoculação. Aplicados após sete dias, apenas azoxystrobin, tebuconazole e epoxiconazole + pyraclostrobin mantiveram o efeito curativo. Aos 10 dias depois da inoculação, os fungicidas reduziram a infecção, mas não controlaram totalmente a doença. Além do efeito curativo, azoxystrobin e triadimenol proporcionaram efeito protetor quando aplicados até 21 dias antes da inoculação de P. psidii. Avaliou-se o tempo mínimo de absorção de azoxystrobin, tebuconazole, triadimenol e trifloxystrobin. Esses quatro fungicidas foram absorvidos pela planta em todos os intervalos testados - 30, 60, 90 e $120 \mathrm{~min}$ - e inibiram a infecção de $P$. psidii em mudas de eucalipto.
\end{abstract}

Palavras-chave: Puccinia psidii, estrobilurinas e triazol.

\section{EFFICACY OF SYSTEMIC FUNGICIDES IN CONTROLLING EUCALYPTUS RUST}

\begin{abstract}
Curative and protective effects of systemic fungicides (azoxystrobin, triadmenol, tetraconazole, tebuconazole and the mixture epoxiconazole + pyraclostrobin) against eucalyptus rust were evaluated. To evaluate the curative effect, eucalyptus plants were sprayed with an inoculum suspension $\left(2 \times 10^{4}\right.$ urediniospores $(\mathrm{mL})$ and with each fungicide after 0, 4, 7, or 10 days. For the protective effect, the plants were sprayed with each fungicide and with the inoculum suspension after $0,7,14,21$, or 28 days. The number of pustules/ leaf, sori/foliar area, and urediniospores/sorus was determined on the first two apical leaves. The best curative effect was achieved with azoxystrobin, tebuconazole, epoxiconazole + pyraclostrobin, and triadimenol, since the number of sori/foliar area and urediniospores/sorus was reduced when applied up to 7 days after inoculation. Regarding protective effect, azoxystrobin and triadimenol were the most effective fungicides, as no pustules formed on the leaves up to 21 days after spray. Azoxystrobin, tebuconazole, triadmenol and trifloxystrobin were absorbed within 30 min after application and no P. psidii infection was detected.
\end{abstract}

Keywords: Puccinia psidii, strobilurins and triazol.

\footnotetext{
${ }^{1}$ Recebido em 30.10.2006 e aceito para publicação em 22.08.2008.

${ }^{2}$ Departamento de Fitopatologia da Universidade Federal de Viçosa (UFV).E-mail: <aalfenas@ufv.br> e < edivalzauza@ suzano.com.br>.
} 


\section{INTRODUÇÃO}

O eucalipto é a essência florestal mais plantada no Brasil, com aproximadamente 3,5 milhões de hectares. A possibilidade do uso múltiplo da madeira: papel e celulose, chapas de aglomerados e de fibras, laminados, compensados, construção civil, carvão vegetal e produção de móveis tornou o eucalipto de grande importância no contexto mundial (SIMÕES et al., 1980).

$\mathrm{O}$ aumento da demanda nos mercados interno e externo dos subprodutos da madeira do eucalipto levou à expansão das áreas plantadas, principalmente em Minas Gerais, no Espírito Santo e Sul da Bahia. Tal fato representa um risco para o surgimento de problemas fitossanitários, sobretudo áreas com condições climáticas favoráveis a doenças e insetos-pragas. A ferrugem das mirtáceas, causada pelo fungo Puccinia psidii Winter, é uma das doenças mais importantes do eucalipto.

O fungo Puccinia psidii foi descrito no Brasil em 1944, incidindo em mudas de Corymbia citriodora (JOFFILY, 1944) no Município de Itaguaí, Rio de Janeiro. No entanto, o primeiro registro de ocorrência do fungo no país foi em mudas de Psidium pomiferum L. (= Psidium guajava L.), por Winter (MACLACHLAM, 1934). Em 1974, cerca de 400.000 mudas de Eucalyptus grandis Hill ex Maiden, de procedência da África do Sul, foram refugadas em decorrência dessa doença(FERREIRA, 1983). Em 1980, a ferrugem incidiu, severamente, em mais de 300 ha de E. grandis com 6 meses de idade, no Vale do Rio Doce, Minas Gerais (FERREIRA e SILVA, 1982).

A doença incide em jardins e minijardins clonais, plantios novos com até 2 anos de idade e brotações no campo após o corte raso. O patógeno afeta órgãos em desenvolvimento como folhas, botões florais, frutos e ramos e causa deformações, minicancros, perda da dominância apical e, provavelmente, redução do crescimento, sendo a presença de pústulas de coloração amarela intensa a característica mais marcante para diagnóstico da doença (ALFENAS et al., 2004). Além do eucalipto, $P$. psidii infecta espécies de vários gêneros de Myrtaceae, incluindo Angophora, Callistemon, Corymbia, Eugenia, Marlierea, Melaleuca, Myrcia, Myrciaria, Pimenta, Psidium, Syzigium e por inoculação sob condições controladas e infectou Heteropyxis natalensis (Heteropyxidaceae) (ALFENAS et al., 2005).

A ferrugem pode ser controlada por resistência inter ou intra-específica (COUTINHO et al., 1998), e em casos emergenciais fungicidas podem ser usados em jardins e minijardins clonais, em plantas jovens ou brotações (RUIZ e ALFENAS, 1989; ALFENAS et al., 2004). O rápido crescimento do eucalipto e a capacidade do patógeno de infectar, apenas, órgãos em estádio juvenil tornam necessária a escolha do fungicida que passará a proteger os lançamentos vegetativos emitidos após a aplicação do produto (DEMUNER e ALFENAS, 1991).

Desde a década de 1980, o triadimenol é o fungicida mais utilizado para o controle da ferrugem (RUIZ et al., 1989). Entretanto, em vista da preocupação quanto ao uso contínuo de um mesmo princípio ativo, aliada às exigências dos órgãos de certificação florestal sobre o uso de produtos que não contenham cloro na sua molécula, pois este, se presente, pode persistir no ambiente, sendo necessária a busca de outros fungicidas eficientes no controle da doença.

No mercado há vários fungicidas eficientes para o controle de ferrugens, como as estrobilurinas e os triazóis (NARUZAWA et al., 2006; GODOY e CANTERI, 2004; MUELLER etal., 2004; CHALFOUN e CARVALHO, 1999). As estrobilurinas têm atividade biológica variada e potencial de controle de ampla gama de fungos. Inibem a respiração mitocondrial ao bloquearem a transferência de elétrons entre os citocromos B e C, o que interfere na formação de ATP(KOELLER, 1998; YPEMAe GOLD, 1999), atuando nos estádios de pré-penetração e inibindo a germinação de esporos, o desenvolvimento de tubos germinativos e a formação de apressórios. Apresentam, também, ação curativa, por inibirem o desenvolvimento do fungo nos estágios pós-germinação e causarem o colapso do micélio dentro do tecido colonizado, bem como ação antiesporulante (VENÂNCIO et al., 1999). Ademais, as estrobilurinas podem induzir alterações fisiológicas em várias culturas e levar ao acréscimo da produtividade (BERTELSEN e SMEDEGAARDPETERSEN, 1998), aumento da tonalidade da cor verde das folhas e atraso da senescência e elevação na concentração de clorofila, proteínas e biomassa, o que favorece o índice de colheita (HABERMEYER et al., 1998; VENÂNCIO et al., 1999). As moléculas desses compostos têm difusão translaminar e decomposição rápida no ambiente são absorvidas pelas folhas de forma gradual e constante, o que confere proteção mais prolongada na superfície (VENÂNCIO et al., 1999). 
A maioria dos fungicidas do grupo dos triazóis tem ação sistêmica acropetal, inibindo a biossíntese de esteróis, especialmente do ergosterol. A deficiência desse esterol e o acúmulo de compostos intermediários induzem a formação de membranas alternativas e a desorganização celular (FORCELINI et al., 2001). Tem efeito fungitóxico elevado e penetração e translocação rápida nos tecidos vegetais, o que evita perda por lixiviação, efeito residual prolongado, e age como protetor nos eventos pré-penetração e como curativo nos eventos pós-penetração (FORCELINI, 1994).

Este trabalho objetivou avaliar o uso de fungicidas sistêmicos dos grupos das estrobilurinas (azoxystrobin e pyraclostrobin) e dos triazóis (triadimenol, tetraconazole, tebuconazole e epoxiconazole) no controle da ferrugem do eucalipto.

\section{MATERIAL E MÉTODOS}

\subsection{Efeito protetor e curativo de fungicidas sistêmicos}

Transplantaram-se mudas de um clone híbrido de Eucalyptus grandis $\mathrm{x}$ Eucalyptus urophylla para vasos com mistura solo: esterco de curral $(2,5: 1)$, previamente esterilizada com brometo de metila $\left(80 \mathrm{cc} / \mathrm{m}^{3}\right)$. As plantas, mantidas em casa de vegetação, semanalmente eram adubadas com formulação comercial Ouro Verde $\AA$, 15 $\mathrm{mL} / \mathrm{L} / \mathrm{vaso}$. Efetuaram-se podas sucessivas para manter as plantas com $20-30 \mathrm{~cm}$ de altura e induzir lançamentos vegetativos.

Nas inoculações, utilizou-se um isolado monopustular de $P$. psidii, UFV-2* raça 1 , obtido de plantas de eucalipto infectadas naturalmente, na região de Itapetininga, SP. Urediniósporos foram pincelados em jambeiros, os quais, depois de mantidos em câmara de nevoeiro e no escuro por $24 \mathrm{~h}$, foram acondicionados em câmara de crescimento a $22^{\circ} \mathrm{C}$ e fotoperíodo de 12 h. Após 12 dias da inoculação, os urediniósporos produzidos foram coletados com um pincel de cerdas macias e armazenados a $-80^{\circ} \mathrm{C}$. Para preparar a suspensão de inóculo, urediniósporos foram transferidos para tubos de ensaio, contendo água mais Tween 20 a 0,05\%, foram homogeneizados em agitador tipo Vortex por 5 min e, em contador automático de partículas (Beckman Coulderâ), calibrou-se a suspensão para a concentração de $2 \times 10^{4}$ urediniósporos $/ \mathrm{mL}$. Para melhor homogeneização, agitou-se a suspensão por $3 \mathrm{~min}$, em agitador magnético (Fisatom modelo 752A). As inoculações foram realizadas com um atomizador De
Vilbbis $\mathrm{n}^{\circ} 15$, pulverizando-se as plantas até o ponto de escorrimento.

Testaram-se os efeitos curativo e protetor dos fungicidas azoxystrobin $0,1 \mathrm{~g}$ i.a./L, triadimenol 0,5 g i.a./L, tetraconazole $0,2 \mathrm{~g}$ i.a./L, tebuconazole 0,4 g i.a./L e epoxiconazole + pyraclostrobin 0,4 g i.a./L, veiculados em água mais $0,02 \%$ de Triton X-100. A dose foi definida por meio da recomendação de rótulo do produto para grandes culturas e, ou, para controle de outras ferrugens.

O efeito curativo foi avaliado após a atomização da suspensão de $2 \times 10^{4}$ urediniósporos $/ \mathrm{mL}$ nas plantas, as quais foram transferidas para câmara de nevoeiro, no escuro, a $24{ }^{\circ} \mathrm{C}$. Após $24 \mathrm{~h}$, foram transportadas para câmara de crescimento a $23^{\circ} \mathrm{Ce} 12 \mathrm{~h}$ de fotoperíodo (RUIZ et al., 1989). Realizaram-se quatro tratamentos, aplicando-se os fungicidas aos 0, 4, 7 e 10 dias após a inoculação. Para avaliar o efeito protetor, foram realizados cinco tratamentos, pulverizando-se os fungicidas nas plantas, e, após $0,7,14,21$ e 28 dias, inoculou-se $P$. psidii. Adotaram-se procedimentos pós-inoculação similares ao do efeito curativo. Em ambos os efeitos, plantas inoculadas e não-tratadas com fungicida e plantas atomizadas com água foram, respectivamente, o controle positivo e negativo.

Após 12 dias da inoculação, constou-se o número de pústulas/folha, de soros/área foliar $\left(1,13 \mathrm{~cm}^{2}=1,2\right.$ cm de diâmetro) e de urediniósporos/soro/área foliar nas duas primeiras folhas apicais completamente expandidas. Utilizaram-se para contar o número de soros, retiraram-se dois discos na porção mediana do limbo foliar, um de cada lado da nervura principal, perfazendo um total de 12 repetições por tratamento ( 3 plantas $\mathrm{x} 2$ folhas $\mathrm{x} 2$ discos). Para determinar o número de urediniósporos/soro, os discos coletados por folha foram inseridos em tubos de ensaio contendo $3 \mathrm{~mL}$ de água mais Tween $20(0,5 \%)$ e agitados em vórtex por $5 \mathrm{~min}$. Finalizada essa etapa, efetuaram-se leituras no contador automático de esporos, cujos dados foram convertidos em porcentagem de inibição de produção de pústulas (IP), porcentagem de inibição de produção de soros (IS) e porcentagem de inibição de produção de esporos (IE), em relação ao controle positivo.

Em um terceiro experimento, determinou-se o tempo mínimo de absorção de azoxystrobin, tebuconazole, triadimenol e trifloxystrobin. Pulverizaram-se os fungicidas

R. Árvore, Viçosa-MG, v.32, n.5, p.829-835, 2008 
em plantas clonais de eucalipto, as quais foram lavadas em água corrente após 0, 30, 60, 90 e 120 min da pulverização, com o auxílio de um chumaço de algodão. Após secagem em condições ambientes, foram inoculadas com uma suspensão de $2 \times 10^{4}$ urediniósporos/ $\mathrm{mL}$ e mantidas sob condições ideais de infecção, conforme relatado anteriormente. Após 12 dias da inoculação, avaliou-se o total da produção de pústulas (IP), da produção de soros (IS) e da produção de esporos (IE). Plantas não-tratadas e tratadas com fungicidas, mas não lavadas, constituíram os controles positivo e negativo, respectivamente.

Cada experimento, realizado em delineamento experimental inteiramente casualizado, foi conduzido por duas vezes. Submeteram-se os resultados à análise de variância e, quando necessário, compararam-se as médias pelo teste de Tukey $(P<0,05)$, com o programa SAS v. 9.1. (SAS Institute, Cary, NC).

\section{RESULTADOS}

\subsection{Efeitos curativo e protetor de fungicidas sistêmicos}

Por meio da análise de variância, não se detectou efeito significativo entre ensaios, sendo os dados analisados estatisticamente com base nas médias dos dois ensaios.

O efeito curativo variou com a época de aplicação com $100 \%$ de controle da ferrugem em todos os fungicidas, aos quatro dias após a inoculação. Com sete dias, os fungicidas azoxystrobin, tebuconazole e pyraclostrobin + epoxiconazole apresentaram $100 \%$ de controle, mas nenhum fungicida inibiu a infecção em $100 \%$ aos 10 dias da inoculação. Os índices de inibição foram melhores com os fungicidas azoxystrobin e tetraconazole, sem diferença estatística (Quadro 1).

Quanto ao efeito protetor, todos os fungicidas aplicados até sete dias antes da inoculação mostraram controle total da doença, mas apenas o azoxystrobin e o triadimenol apresentaram controle de $100 \%$ quando aplicados aos 14 ou 21 dias antes da inoculação. Aos 28 dias, nenhum dos fungicidas preveniu a infecção em $100 \%$, apesar da alta inibição com triadimenol e azoxystrobin (Quadro 2).

$\mathrm{Na}$ análise de variância do terceiro ensaio, não houve efeito da interação fungicida: período de avaliação (horas em pós-inoculação) (dados não apresentados). Com todos os fungicidas e tempos de absorção testados, ocorreu inibição total da produção de pústulas (IP), da produção de soros (IS) e da produção de esporos (IE). Assim, os fungicidas foram absorvidos pelas folhas num período de tempo de 30 min e exerceram ação sobre a infecção e esporulação.

As porcentagens de inibição de produção de pústulas, de produção de soros e de produção de esporos apresentaram correlação significativa (Quadro 3), demonstrando que apenas uma dessas variáveis deve ser utilizada nas avaliações.

\section{DISCUSSÃO}

O efeito curativo significativo dos fungicidas aplicados até sete dias da inoculação de $P$. psidii possibilita estabelecer melhores intervalos de aplicação desses produtos. Fungicidas com efeito curativo inibem o desenvolvimento do haustório e, ou, crescimento micelial no interior dos tecidos (FORCELINI, 1994).

Quadro 1 - Efeito curativo de fungicidas sistêmicos no controle da ferrugem do eucalipto em pulverizações aos $0,4,7$ e 10 dias antes da inoculação de Puccinia psidii

Table 1 - Curative effect of systemic fungicides on the control of eucalyptus rust sprayed at 0, 4, 7, and 10 days after inoculation of Puccinia psidii

\begin{tabular}{|c|c|c|c|c|c|c|}
\hline \multirow[t]{3}{*}{ Fungicida } & \multicolumn{6}{|c|}{ Época de aplicação (dias após a inoculação) } \\
\hline & \multicolumn{3}{|c|}{7 dias } & \multicolumn{3}{|c|}{10 dias } \\
\hline & IP $(\%)$ & IS $(\%)$ & IE $(\%)$ & IP $(\%)$ & IS $(\%)$ & IE $(\%)$ \\
\hline$\overline{\text { Azoxystrobin }}$ & $100 \mathrm{a}^{*}$ & $100 \mathrm{a}$ & $100 \mathrm{a}$ & $86 \mathrm{a}$ & $91 \mathrm{a}$ & $95 \mathrm{a}$ \\
\hline Tebuconazole & $100 \mathrm{a}$ & $100 \mathrm{a}$ & $100 \mathrm{a}$ & $74 \mathrm{ab}$ & $81 \mathrm{a}$ & $71 \mathrm{~b}$ \\
\hline Pyraclostrobin-epoxiconazole & $100 \mathrm{a}$ & $100 \mathrm{a}$ & $100 \mathrm{a}$ & $46 \mathrm{~b}$ & $61 \mathrm{~b}$ & $72 \mathrm{~b}$ \\
\hline Tetraconazole & $95 \mathrm{~b}$ & $97 \mathrm{~b}$ & $96 \mathrm{~b}$ & $87 \mathrm{a}$ & 86 a & $85 \mathrm{ab}$ \\
\hline Triadimenol & $96 a b$ & $97 \mathrm{~b}$ & $96 \mathrm{~b}$ & $66 \mathrm{ab}$ & $69 \mathrm{~b}$ & $83 \mathrm{ab}$ \\
\hline
\end{tabular}

-Em cada coluna, as médias seguidas da mesma letra não diferem entre si (teste de Tukey, $P=0,01$ ).

IP = porcentagem de inibição de produção de pústulas; percentage inhibition of pustule production. IS = porcentagem de soros; percentage of sori. IE = porcentagem de esporos; percentage of spores. 
Quadro 2 - Efeito protetor de fungicidas sistêmicos no controle da ferrugem do eucalipto aplicados aos 0, 4, 7 e 10 dias antes da inoculação de Puccinia psidii

Table 2 - Protective e effect of systemic fungicides on the rust control at 0, 4, 7, and 10 days before inoculation of Puccinia psidii

\begin{tabular}{|c|c|c|c|c|c|c|c|c|c|}
\hline \multirow[t]{3}{*}{ Fungicida } & \multicolumn{9}{|c|}{ Época de aplicação (dias antes da inoculação) } \\
\hline & \multicolumn{3}{|c|}{14 dias } & \multicolumn{3}{|c|}{21 dias } & \multicolumn{3}{|c|}{28 dias } \\
\hline & IP $(\%)$ & IS (\%) & IE $(\%)$ & IP $(\%)$ & IS (\%) & IE $(\%)$ & IP $(\%)$ & IS $(\%)$ & $\overline{I E}(\%)$ \\
\hline Azoxystrobin & $100 \mathrm{a}^{*}$ & $100 \mathrm{a}$ & $100 \mathrm{a}$ & $100 \mathrm{a}$ & $100 \mathrm{a}$ & $100 \mathrm{a}$ & $94 \mathrm{ab}$ & $93 \mathrm{ab}$ & $70 \mathrm{~b}$ \\
\hline Triadimenol & $100 \mathrm{a}$ & $100 \mathrm{a}$ & $100 \mathrm{a}$ & $100 \mathrm{a}$ & $100 \mathrm{a}$ & $100 \mathrm{a}$ & $97 \mathrm{a}$ & $97 \mathrm{a}$ & 96 a \\
\hline Tebuconazole & $98 \mathrm{ab}$ & $96 \mathrm{ab}$ & 99 a & $94 \mathrm{~b}$ & $77 \mathrm{~b}$ & $53 \mathrm{~b}$ & $84 \mathrm{~b}$ & $82 \mathrm{c}$ & $20 \mathrm{c}$ \\
\hline Pyraclostrobin-epoxiconazole & $93 \mathrm{~b}$ & $89 \mathrm{bc}$ & $71 \mathrm{~b}$ & $95 \mathrm{~b}$ & $75 \mathrm{~b}$ & $56 \mathrm{~b}$ & $85 \mathrm{ab}$ & $85 \mathrm{bc}$ & $30 \mathrm{c}$ \\
\hline Tetraconazole & $87 \mathrm{~b}$ & $83 \mathrm{c}$ & $84 \mathrm{ab}$ & $85 \mathrm{c}$ & $75 \mathrm{~b}$ & $57 \mathrm{ab}$ & $16 \mathrm{c}$ & $19 \mathrm{~d}$ & $10 \mathrm{c}$ \\
\hline
\end{tabular}

-Em cada coluna, as médias seguidas da mesma letra não diferem entre si (teste de Tukey, $P=0,01$ ).

IP = porcentagem de inibição de produção de pústulas; percentage inhibition of pustules production. IS = porcentagem de soros; percentage of sori. IE = porcentagem de esporos; percentage of spores.

Quadro 3 - Nível de significância do coeficiente de correlação de Pearson entre as porcentagens de inibição de produção de pústulas (IP), soros (IS) e esporos (IE)

Table 3 - Pearson correlation coefficient

\begin{tabular}{|c|c|c|c|c|}
\hline \multirow[t]{3}{*}{ Variável } & \multicolumn{4}{|c|}{ Ensaio } \\
\hline & \multicolumn{2}{|c|}{ Efeito curativo } & \multicolumn{2}{|c|}{ Efeito protetor } \\
\hline & IP & IS & IP & IS \\
\hline IP & - & $\mathrm{r}=0,9875(P \leq 0,0001)$ & - & $\mathrm{r}=0,9458(P \leq 0,0001)$ \\
\hline IE & $\mathrm{r}=0,8833(P \leq 0,0007)$ & $\mathrm{r}=0,8778(P \leq 0,0008)$ & $\mathrm{r}=0,7036(P \leq 0,0034)$ & $\mathrm{r}=0,7681 \quad(P \leq 0,0008)$ \\
\hline
\end{tabular}

Alguns desses fungicidas obtiveram resultados semelhantes aos deste estudo, com efeito curativo do triadimenol, aplicado até nove dias da inoculação de $P$. psidii em E. grandis (RUIZ et al., 1987). Os triazóis têm como principais características: penetração e translocação rápidas, ação curativa sobre infecções já iniciadas e reduzida perda por lixiviação (FORCELINI, 1994).

As estrobilurinas são um grupo de fungicidas sistêmicos que atuam inibindo a respiração fúngica (WU e TIEDEMANN, 2001). O controle de $100 \%$ com a aplicação de epoxiconazole + pyraclostrobin até sete dias da inoculação possivelmente se deveu pelo aumento do espectro de ação e da duração da atividade antifúngica, proporcionado pela mistura de produtos com modos de ação diferentes (REUVENI, 2001).

A eficiência do azoxystrobin, aplicado até 28 dias antes da inoculação, deveu-se à proteção e redistribuição superficial, a difusão translaminar e a proteção sistêmica do produto. Os fungicidas com ação protetora atuam inibindo a germinação de esporos, durante a formação do tubo germinativo e do apressório (FORCELINI, 1994).
A eficiência do triadimenol foi semelhante quando aplicaram o produto no dia da inoculação de $P$. psidii em $E$. grandis e aos 5 e 10 dias antes desse procedimento (RUIZ et al., 1989) e na concentração de 0,4 e 0,8 g i.a./L desse produto para controle de $P$. psidii em mudas de E. cloeziana com ação protetora total por até 28 dias (DEMUNER e ALFENAS, 1991). Os tratamentos com esse produto, aplicados via pulverizações, reduziram significativamente a incidência da ferrugem em brotações de E. cloeziana em condições de campo (ALFENAS et al., 1993).

Os fungicidas azoxystrobin, tebuconazole, triadimenol e trifloxystrobin foram absorvidos pelas folhas em até $30 \mathrm{~min}$ após a pulverização e inibiram a infecção e esporulação. Resultados semelhantes foram obtidos com os triforine e triadimenol para o controle de $P$. psidii em eucalipto (RUIZ e ALFENAS, 1989) e para a absorção de triadimefon e propiconazole por folhas de cafeeiro 'Catuaí Vermelho' no controle de Hemileia vastatrix (NUNES et al., 1986).

Foi possível estabelecer melhores intervalos de pulverizações dos fungicidas testados e recomendar a alternância de aplicação destes com diferentes princípios

R. Árvore, Viçosa-MG, v.32, n.5, p.829-835, 2008 
ativos. Assim, o manejo integrado da ferrugem pode incluir o uso de fungicidas em viveiros e jardins ou minijardins clonais e, excepcionalmente, em campo para materiais genéticos altamente suscetíveis. Recomendase efetuar uma ou duas pulverizações de azoxystrobin em intervalos quinzenais ao surgirem os primeiros sinais da doença e sob condições favoráveis à infecção e desenvolvimento da doença, como temperaturas entre 18 e $25^{\circ} \mathrm{C}$, períodos superiores a $6 \mathrm{~h}$ de molhamento foliar noturno por cinco dias consecutivos e existência de tecidos juvenis (RUIZ et al., 1989). Persistindo a doença e as condições favoráveis para seu progresso, devem-se alternar as pulverizações com triadimenol em intervalos quinzenais. Esse esquema de pulverizações será eficiente quando existir uma rotina de monitoramento de doenças no viveiro e no campo, para ser possível detectar o início da epidemia. Caso contrário, quando a epidemia já estiver avançada, com alta incidência da doença, devem-se iniciar as pulverizações com triadimenol ou tebuconazole e, após duas pulverizações, alternar com azoxystrobin. No entanto, sempre que possível, fungicidas devem ser usados como parte de um sistema de manejo integrado da doença. Dessa forma, devem-se adotar estratégias de anti-resistência como o uso de dose prescrita pelo fabricante, aplicar as estrobilurinas preventivamente sem exceder 30 a $50 \%$ do total de aplicações e alternar fungicidas com diferentes modos de ação. Devem-se evitar aplicações individuais e repetidas em um mesmo cultivo com os fungicidas inibidores da demetilação (DMIs) como os triazóis e, quando possível, utilizar misturas ou alternância com outros fungicidas para os quais não haja resistência cruzada com os DMIs ou restringir as aplicações nas fases críticas de cultivo (FORCELINI et al., 2001). Contudo, ainda não há um fungicida registrado para a cultura do eucalipto. Assim, deve-se introduzir um novo produto após testes preliminares, para avaliar a fitotoxicidade do fungicida ao material genético que se deseja proteger.

\section{CONCLUSÕES}

- Azoxystrobin, tebuconazole e epoxiconazole + pyraclostrobin, quando aplicados até sete dias após a inoculação de $P$. psidii, proporcionaram controle total da ferrugem.

- Azoxystrobin e triadimenol controlaram totalmente a doença, quando aplicados até 21 dias antes da inoculação.
- Azoxystrobin, tebuconazole, triadimenol e trifloxystrobin foram absorvidos por plantas de eucalipto no intervalo de $30 \mathrm{~min}$ após a aplicação. Todos os fungicidas aplicados imediatamente antes (tempo 0) ou aos quatro dias antes da inoculação ofereceram controle total da ferrugem.

- Azoxystrobin apresentou efeitos curativo e protetor, sendo opção para controle da ferrugem do eucalipto.

- Existe correlação entre os valores das porcentagens de inibição de produção de pústula, de inibição de produção de soros e de produção de esporos.

\section{REFERÊNCIAS}

ALFENAS, A. C. et al. Eficiência de triadimenol, oxicarboxin e diniconazole para o controle da ferrugem (Puccinia psidii) em brotações de Eucalyptus cloeziana, em condições de campo. Revista Árvore, v.17, n. 2, p.247-263, 1993.

Alfenas, A. C. et al. Clonagem e doenças do eucalipto. Viçosa, MG: Universidade Federal de Viçosa, 2004. 442p.

ALFENAS, A. C. et al. Heteropyxis natalensis, a new host of Puccinia psidii rust. Australasian Plant Pathology, v.34, p.285-286, 2005.

BERTELSEN, J. R.; SMEDEGAARD-PETERSEN, V. Effect of the strobilurin fungicide azoxystrobin on leaf sprophytes, host physiology and yield of wheat crops. In: INTERNATIONAL CONGRESS OF PLANT PATHOLOGY, 7., 1998, Glasgow. Abstract... Glasgow: Br. Soc. Plant Pathol., 5.6.1. 1998.

CHALFOUN, S. M.; CARVALHO, V. L. Controle químico da ferrugem (Hemileia vastatrix berk \& br.) do cafeeiro através de diferentes esquemas de aplicação. Pesquisa Agropecuária Brasileira, v.34, n.3, p.363-367, 1999.

COUTINHO, T. A. et al. Eucalyptus rust: A disease with the potential for serious international implications. Plant Disease, v.82, n.7, p.819-825, 1998.

DEMUNER, L. N.; ALFENAS, A. C. Fungicidas sistêmicos para o controle da ferrugem causada por Puccinia psidii em Eucalyptus cloeziana. Fitopatologia Brasileira, v.16, n.3, p.174-176, 1991. 
FERREIRA, F. A. Ferrugem do eucalipto. Revista Árvore, v.7, n.1, p.91-109, 1983.

FERREIRA, F. A.; SILVA, A. R. Comportamento de procedências de Eucalyptus grandis e de Eucalyptus saligna a ferrugem (Puccinia psidii). Fitopatologia Brasileira, v.7, n.1, p.23-8, 1982.

FORCELLINI, C. A. Fungicidas inibidores da síntese de esteróis. I. Tiazoles. Revisão Anual de Patologia de Plantas, v.2, p.335-355, 1994.

FORCELLINI, C. A.; GOELLNER, C. I.; MAY-DEMIO, L. L. Resistência de fungos a fungicidas. Revisão Anual de Patologia de Plantas, v.9, p.339-381, 2001.

GODOY, C. V.; CANTERI, M. G. Efeitos protetor, curativo e erradicante de fungicidas no controle da ferrugem da soja causada por Phakopsora pachyrhizi, em casa de vegetação. Fitopatologia Brasileira, v.29, n.1, p.97-101, 2004.

HABERMEYER, J.; GERHARD, M.; ZINKERNAGEL, V. The impact of strobilurins on the plant physiology of wheat. In: INTERNATIONAL CONGRESS OF PLANT PATHOLOGY, 7., 1998, Glasgow. Abstract... Glasgow: Br. Soc. Plant Pathol., 5.6.3. 1998.

JOFFILY, J. Ferrugem do eucalipto. Bragantia, v.4, n.8, p.475-487, 1944.

KOELLER, W. Mode of action of fungicides in real plant pathogens. . In: VII INTERNATIONAL CONGRESS OF PLANT PATHOLOGY, 7., 1998, Glasgow. Abstract... Glasgow: Br. Soc. Plant Pathol., 5.5.1S, 1998.

MACLACHLAN, J. D. A rust of pimento tree in Jamaica. Phytopatology, v. 28, n. 8 , p.157-170, 1934.

MUELLER, D. S.; JEFFERS, S. N.; BUCK, J. W. Effect of timing of fungicide applications on development of rusts on daylily, geranium, and sunflower. Plant Disease, v.88, n.6, p.657-661, 2004.
NARUZAWA, E. S. et al. Estudos epidemiológicos e controle químico de Phakopsora euvitis. Fitopatologia Brasileira, v.31, n.1, p.41-45, 2006.

NUNES, A. M. L.; ZAMBOLIM, L.; VALE, F. X. R. Bioensaio para a determinação do tempo de penetração de fungicidas sistêmicos em folhas de cafeeiro. Fitopatologia Brasileira, v.11, p.374, 1986. Suplemento.

REUVENI, M. Improved control of powdery mildew (Spharatheca pannosa) of nectarines in Israel using strobilurin and polyoxin B fungicides; mixtures with sulfur; and early bloom applications. Crop Protection, v.20, n.8, p.663-668, 2001.

RUIZ, R. A. R.; ALFENAS, A. C. Absorção e translocação de fungicidas sistêmicos em Eucalyptus grandis para o controle da ferrugem do eucalipto, causada por Puccinia psidii. Fitopatologia Brasileira, v.14, n.1, p.47-50, 1989.

RUIZ, R. A. R. et al. Fungicidas protetores e sistêmicos para o controle da ferrugem do eucalipto causada por Puccinia psidii. Revista Árvore, v.11, n.1, p.56-65, 1987.

SIMÕES, J. W. et al. Crescimento e produção de madeira de eucalipto. IPEF, v.20, p.77-97, 1980.

VENÂNCIO, W. S. et al. Novos fungicidas. IProdutos naturais e derivados sintéticos: estrobilurinas e fenilpirroles. Revisão Anual de Patologia de Plantas, v.7, p.103-155, 1999.

YPEMA, H. L.; GOLD, R. E. Kresoxim-methyl: modification of a naturally occurring compound to produce a new fungicide. Plant Disease, v.83, n.1, p.4-19, 1999.

WU, Y.; TIEDEMANN, A. V. Physiological effects of azoxystrobin and epoxiconazole on senescence and the oxidative saturs of wheat. Pesticide Biochemistry and Physiology, v.71, n.1, p.1-10, 2001. 
